\title{
Correction to: Candesartan treatment enhances liposome penetration and anti-tumor effect via depletion of tumor stroma and normalization of tumor vessel
}

\author{
Xin-jun Cai ${ }^{1}$ - Zeng Wang ${ }^{2} \cdot$ Ying-ying Xu ${ }^{1} \cdot$ Gao-yi Yang ${ }^{3} \cdot$ Ruo-ying Zhang ${ }^{1} \cdot$ Yu Wang ${ }^{1}$ \\ Published online: 26 August 2020 \\ (C) Controlled Release Society 2020
}

\section{Correction to: Drug Delivery and Translational Research} https://doi.org/10.1007/s13346-020-00842-0

In the original article there is an error in part $\mathbf{a}$ in the caption of Fig. 6. Following is the complete corrected caption:

Fig. 6 Effect of different treatment groups dosing regimens on the percentage of CEPCs in PBMCs. a The representative photographs of flow cytometry analysis for the determination of the percentage of $\mathrm{CD} 45^{-} \mathrm{CD} 146^{+}$cells in PBMCs. A1: Control group. A2: Candesartan group $(10 \mathrm{mg} / \mathrm{kg})$. A3: Candesartan group $(20 \mathrm{mg} / \mathrm{kg})$. b The percentage of CEPCs in PBMCs after various treatment. Results were expressed as mean \pm standard deviation (SD) $(n=5)$. Statistical comparisons were carried out by using one-way ANOVA. Compared with control group: $p<0.05$; $p<0.01$;

$p<0.001$

Publisher's note Springer Nature remains neutral with regard to jurisdictional claims in published maps and institutional affiliations.

The online version of the original article can be found at https://doi.org/ $10.1007 / \mathrm{s} 13346-020-00842-0$

Xin-jun Cai

zjtcmcxj@zcmu.edu.cn

1 Department of Pharmacy, Zhejiang Hospital of Integrated Traditional Chinese and Western Medicine, Hangzhou Red Cross Hospital, Hangzhou 310003, Zhejiang, People's Republic of China

2 Department of Pharmacy, Cancer Hospital of the University of Chinese Academy of Sciences (Zhejiang Cancer Hospital), Institute of Cancer and Basic Medicine (ICBM), Chinese Academy of Sciences, Hangzhou 310022, China

3 Department of Ultrasound, Zhejiang Hospital of Integrated Traditional Chinese and Western Medicine, Hangzhou Red Cross Hospital, Hangzhou 310003, Zhejiang, People's Republic of China 